\title{
Factores que contribuyen al incremento de infectados covid-19 en las diferentes familias de Latinoamérica
}

Factors contributing to the increase of covid-19-infected patients in different families in Latin America

Vilma Amparo Junchaya Yllescas ${ }^{1, a}$, Diana Esmeralda Andamayo Flores ${ }^{1, b}$, G. Moran Ávila 1,c, C. Gonzales Melchor ${ }^{1, \mathrm{c}}$.

\section{RESUMEN}

Objetivo: Evaluar los factores que contribuyen al incremento de pacientes infectados por el COVID-19 en las diferentes familias de Latinoamérica, siendo el Materialy Métodos: Un estudio descriptivo transversal. La población consistió en usuarios de las redes sociales con respecto a factores que contribuyen al contagio de la COVID-19. Se utilizó un cuestionario digital con variables sociodemográficas, factores bioseguridad, factores culturales y factores ambientales. Se realizó análisis descriptivo y bivariado según la naturaleza de las variables. Resultados: Luego de analizar 1123 cuestionarios de usuarios del Facebook de Latinoamérica, que incluyeron: Argentina, Bolivia, Brazil, Chile, Colombia, Ecuador, Paraguay, Uruguay, Venezuela y Perú, el $77 \%$ fueron mujeres siendo el $81 \%$ de 18 a 31 años. Sobre los factores de bioseguridad que contribuyen al contagio de la población responde un $43 \%$ señala se debe por no emplear mascarilla y protector facial, así también como factores culturales un $55 \%$ indica que no interesa respetar normas, como factores ambientales un $74 \%$ considera que la contaminación ambiental tiene que ver con el incremento, otro $59 \%$ responde que la población de zona de vivienda urbana es la más contagiada y un $49 \%$, refiere que el aire es el elemento contaminante que contribuye al contagio de la COVID-19. Conclusiones: Los usuarios del Facebook consideran que los pobladores de Latinoamérica se contagian por no cumplir factores de bioseguridad, por factores culturales, por diversos factores ambientales, cuyas condiciones que viven muchas las personas los vuelven aún vulnerables y mayor peligro.

PALABRAS CLAVE: Pacientes infectados, Covid-19, familias de Latinoamérica.

\section{SUMMARY}

Objective: To evaluate the factors that contribute to the increase of patients infected by COVID-19 in different families in Latin America. Material and methods: Being a cross-sectional descriptive study. The population consisted of users of social networks with respect to factors contributing to COVID-19 infection. A digital questionnaire was used with sociodemographic variables, biosecurity factors, cultural factors and environmental factors. Descriptive and bivariate analysis was performed according to the nature of the variables. Results:1123 questionnaires of Facebook users from Latin America were analyzed, including: Argentina, Bolivia, Brazil, Chile, Colombia, Ecuador, Paraguay, Uruguay, Venezuela and Peru, most of them women (77\%), (81 \%) between 18 and 31 years old. About the biosecurity factors that contribute to the contagion of the population, $43 \%$ of the

Escuela Profesional de Ciencias Farmacéuticas y Bioquímica, Universidad Privada de Huancayo Franklin Roosevelt. Huancayo, Perú.

a Docente. ORCID ID: https://orcid.org/0000-0002-5035-2157

b Docente. ORCID ID: https://orcid.org/0000-0003-3357-3537

Estudiante. 
respondents indicated that it is due to not using masks and face shields, as well as cultural factors, $55 \%$ indicated that it is not interested in respecting norms, as environmental factors, $74 \%$ considered that environmental pollution has to do with the increase, another $59 \%$ responded that the population of urban housing areas is the most infected and $49 \%$ said that the air is the polluting element that contributes to the contagion of COVID-19. Conclusions: Facebook users consider that Latin American inhabitants are infected by not complying with biosecurity factors, cultural factors, and various environmental factors, whose conditions that many people live in make them even more vulnerable and more dangerous.

Key words: Infected patients, Covid-19, Latin American families.

\section{INTRODUCCIÓN}

La enfermedad por el nuevo coronavirus (COVID-19) causada por el virus SARS-CoV-2, fue detectada por primera vez durante la epidemia del distrito de Wuhan en la provincia de Hubei en China en diciembre de 2019. Desde entonces ha tenido una rápida y exitosa expansión en países asiáticos y europeos, debido principalmente a la demora en la toma de decisiones drásticas para contenerlo. Y es que a pesar de ser la primera vez con que disponemos con tanta información en tiempo real de una Pandemia, al parecer el exceso de la misma ha generado diversas interpretaciones, lo que no ha permitido un análisis oportuno de este problema en los países más afectados (1). Por ello, y ante el surgimiento de casos en Latinoamérica se debe tomar como experiencia lo sucedido en estos continentes y reconocer los desafíos a los cuales se enfrenta esta región ante la inminente expansión de este virus en esta región. Si bien es cierto el coronavirus SARS-CoV-2 no afecta a todos por igual, no obstante, se encuentra como posibles factores de contagio, las personas adultas mayores, igualmente que afecta más a los hombres que a las mujeres, así también a niños y jóvenes, donde la enfermedad se da de manera más leve o asintomática (2). Algunos estudios refieren que la incidencia y gravedad se puede relacionar con la presencia de enfermedades crónicas, de tal forma que las comorbilidades pueden explicar buena parte de estas diferencias por edad y sexo; también hay que tener en cuenta otros posibles factores, como el tabaquismo, la obesidad, el funcionamiento del sistema inmunológico o la genética y que la población socialmente más vulnerable puede verse más afectada por esta pandemia (3).

Por otro lado, el incremento de infectados por el COVID-19, a nivel de Latinoamérica es alarmante, por tanto hasta el 29 de junio de 2021, un total de 37.208.956 casos de COVID-19 han sido registrados en América Latina y el Caribe. Brasil es el país más afectado por esta pandemia en la región, con alrededor de 18,4 millones de casos confirmados. Argentina se ubica en segundo lugar, con aproximadamente 4,4 millones de infectados. México, por su parte, ha registrado un total de 2507453 casos; dentro de los países más afectados por el nuevo tipo de coronavirus en América Latina también se encuentran Colombia, Perú, Chile y Ecuador (4).

El presente trabajo tuvo como objetivo evaluar los factores que contribuyen al incremento de pacientes infectados por el COVID-19 en las diferentes familias de Latinoamérica.

\section{MATERIAL Y MÉTODOS}

Se realizó un estudio transversal en los usuarios del facebook, entre el 15 de Marzo a 15 de Abril 2021. La población diana fueron 1123 usuarios mayores de edad de países de Latinoamérica, quienes a partir de un fanpage creado como página especialmente para ser un canal de comunicación, donde se invitaba a registrar el cuestionario. El instrumento utilizado fue un cuestionario digital. Para validar el instrumento, se utilizó la técnica Delphi en 6 expertos pertenecientes a diferentes profesiones de salud, quienes valoraron la idoneidad de cada uno de los ítems mediante una puntuación de 1 a 5 puntos en escala de Likert, donde 1 representó valores muy deficiente y 5 valores de muy bueno. El cuestionario tuvo 9 preguntas, dividas en 4 secciones: Datos sociodemográficos (4 preguntas), Factores bioseguridad (1 pregunta), factores culturales (1 pregunta) factores ambientales (3 preguntas). El cuestionario virtual fue creado en un formulario de Google y difundido a través de las redes sociales. Se solicitó el consentimiento informado de cada participante, lo que garantizó su anonimato en todo momento. El análisis estadístico se realizó con frecuencias y porcentajes. De una población aproximada de 23000000 de usuarios se calculó un tamaño de muestra de 1089 para que los datos fueran significativos al 95\%, con un error muestral del 5\%. Finalmente se obtuvieron 1123 encuestas, 
lo que nos permitió un análisis estadístico con un error muestral menor del $2 \%$, para un nivel de confianza del $98 \%$. Este estudio cuenta con la aprobación del Comité de Ética de la Investigación de la Universidad Privada de Huancayo Franklin Roosevelt.

\section{RESULTADOS}

Se recibieron 1123 encuestas correctamente llenadas, de países de Latinoamérica como Argentina, Bolivia, Brazil, Chile, Colombia, Ecuador, Paraguay, Uruguay, Venezuela y Perú. Siendo sus características sociodemográficas que gran parte fueron mujeres (77\%), más de la mitad (81\%) tenían entre 18 y 31 años. La mayoría (69 \%) eran de grado de instrucción superior, de ocupación profesional en el $45 \%$, un $42 \%$ no cuenta con sueldo fijo, $63 \%$ de clase media.

Tabla 1. ¿Qué factores de bioseguridad de la población cree usted que contribuye al contagio de la COVID-19?

\begin{tabular}{lcc}
\hline \multicolumn{1}{c}{ Factores Bioseguridad } & Frecuencia & $\mathbf{\%}$ \\
\hline Lavado De Manos & 185 & $16 \%$ \\
Mascarilla & 138 & $12 \%$ \\
Guantes & 5 & $0 \%$ \\
Protector Facial & 105 & $9 \%$ \\
No Aplica Distanciamiento Social & 234 & $21 \%$ \\
Mascarilla Y Protector Facial & 456 & $41 \%$ \\
Total & 1123 & $100 \%$ \\
\hline
\end{tabular}

Fuente: Cuestionario digital Fanpage 15 de Marzo a 15 de Abril 2021.

Tabla 2. ¿Qué factores culturales de la población cree usted que contribuye al contagio de la COVID-19?

\begin{tabular}{lcc}
\hline Factores Culturales & Frecuencia & $\mathbf{\%}$ \\
\hline Aglomeración De Personas & 266 & $24 \%$ \\
Falta De Empatía & 234 & $21 \%$ \\
No Interesa Respetar Normas & 623 & $55 \%$ \\
Total & 1123 & $100 \%$ \\
\hline Fuente: Cuestionario digital Fanpage 15 de Marzo a 15 de \\
Abril 2021.
\end{tabular}

Tabla 3. ¿Considera usted que la contaminación ambiental tiene que ver con el aumento del COVID - 19?

\begin{tabular}{lcc}
\hline Factores ambientales & Frecuencia & Porcentaje \\
\hline $\mathrm{Si}$ & 828 & $74 \%$ \\
$\mathrm{No}$ & 295 & $26 \%$ \\
Total & 1123 & $100 \%$ \\
\hline Fuente: Cuestionario digital Fanpage 15 de Marzo a 15 de \\
Abril 2021.
\end{tabular}

En relación con la percepción de los usuarios del Facebook, sobre los factores de bioseguridad que contribuyeron al contagio de la población en 43 $\%$, se debe por no emplear mascarilla y protector facial (tabla 1), así también como factores culturales un $55 \%$ respondieron porque no interesa respetar normas (tabla 2), como factores ambientales un $74 \%$ considera que la contaminación ambiental tiene que ver con el aumento del COVID - 19 (tabla 3), otro 59 $\%$ señalaron que la población de zona de vivienda urbana es la que más se contagia de la COVID - 19 (tabla 4) y un $49 \%$ considera que el aire es el elemento contaminante que contribuye al contagio de la COVID - 19 en la población (tabla 5).

Tabla 4. ¿Qué zona de vivienda de la población cree usted que padece del contagio de la COVID - 19?

\begin{tabular}{lcc}
\hline Ubicación Vivienda & Frecuencia & Porcentaje \\
\hline Zona Rural & 460 & $41 \%$ \\
Zona Urbana & 663 & $59 \%$ \\
Total & 1123 & $100 \%$ \\
\hline
\end{tabular}

Fuente: Cuestionario digital Fanpage 15 de Marzo a 15 de Abril 2021.

Tabla 5. ¿Qué elemento contaminante de la población cree usted que contribuye al contagio de la COVID 19 ?

\begin{tabular}{lcc}
\hline Elemento Contaminante & Frecuencia & Porcentaje \\
\hline Suelo & 381 & $34 \%$ \\
Agua & 192 & $17 \%$ \\
Aire & 550 & $49 \%$ \\
Total & 1123 & $100 \%$
\end{tabular}

Fuente: Cuestionario digital Fanpage 15 de Marzo a 15 de Abril 2021.

\section{DISCUSIÓN}

En general, los usuarios adultos del Facebook percibe que la población de Latinoamérica no estuvieron aplicando adecuadas normas de bioseguridad para evitar el contagio de la COVID-19, hallándose un $43 \%$ quienes sostienen que no emplean mascarilla y protector facial, cuando Caicoya señala que las mascarillas además de reducir la contaminación del aire y de las superficies inanimadas y que por prudencia se recomendaría guardar al menos $30 \mathrm{~cm}$ de distancia y que la válvula espiratoria solo tiene aplicación en ambientes pulvígenos laborales, nunca en infeccioso; sumado a ello los inconvenientes 
de la mascarilla, como el coste, la incomodidad, el uso inadecuado, la limpieza, el reciclado y sobre todo, que no todo el mundo la tolera ni es utilizable en todas las circunstancias; siendo importante que se continúe promoviendo que la mascarilla, utilizada correctamente, es la estrategia preferente para asegurar la protección de la comunidad (5). En cuanto a los protectores faciales, Armando indica que es muy distinto, gracias a su filtro de nanofibras de polipropileno, garantizado para no dejar pasar microgotículas, aunque no sirve para gases o vapores, a pesar de crear un sello hermético de boca y nariz que incomoda en uso prolongado, y aunque son más caras, se consideran reusables (6).

Otros trabajos señalan los efectos negativos del uso prolongado del mismo cubrebocas o mascarilla, aduciendo que el aliento las humedece y eso favorece la creación de reservorios para diversos microorganismos, por lo que se recomienda usarlas por tiempo limitado o cambiarlas, además de cuidar otras medidas, como no tocarla por el frente al quitársela, proceder a desinfectarla, con varios métodos, sumado a la contaminación registrada en varias playas del mundo con los desechos y con los justos reclamos del personal de salud a nivel nacional e internacional (7). En consecuencia es importante evitar el riesgo de contagio de la población, por ello el utilizar medidas de protección adecuado de acuerdo al nivel de exposición, permitirá disminuir la posibilidad de enfermar de COVID 19 dentro y fuera de su lugar de trabajo o vivienda. En cuanto a que un $16 \%$ respondieron que no utilizan el lavado de manos, cuando Gabriela et al., mencionan que en lavado de manos al emplearse el uso de desinfectante este permite la inactivación del coronavirus SARSCOV-2; así también el Programa sobre Saneamiento e Higiene de la UNICEF, refiere que nuestras manos son la principal herramienta de trabajo porque las utilizamos para un sinfín de actividades, muchos gérmenes, bacterias y virus pueden impregnarse en ellas y al momento de tocarnos la cara, los llevamos a las vías de acceso a nuestro organismo. Es por ello que "un gesto tan simple como lavarse las manos con agua y jabón puede ser clave para la supervivencia de millones de personas, sobre todo de los más pequeños (8).

Si bien es cierto, los usuarios del Facebook de diversos países de Latinoamérica, respondieron como factores culturales de la COVID-19, que un $55 \%$ no les interesa respetar las normas, cuando el estudio de Jara (9), quien demuestra que el incumplimiento de normas por parte de los peruanos influye en el aumento de números de casos por COVID-19 de igual forma, la falta de empatía se vincula con la inconciencia social de los jóvenes debido a que deciden ignorar las normas y prefieren divertirse, poniendo en riesgo a las personas que lo rodean. Asimismo, con la investigacion Pichihua (10), cuando menciona que el coronavirus ha impactado muy fuertemente en países de Latinoamérica como Chile, Perú y Argentina por la falta de respeto en cuanto a las limitaciones a la libertad de los ciudadanos con el objeto de controlar la propagación de aquel. En tanto la percepción de los usuarios resulta asertiva.

La respuesta como factores ambientales, que un $74 \%$ considera que la contaminación ambiental tiene que ver con el aumento del COVID - 19, como el estudio de Arango et al., quienes demostraron que la contaminación ambiental se convirtió en un efecto facilitador de la pandemia pues la transmisión por contacto entre humanos a través de aerosoles representa la vía de contagio, de tal forma que obligó al distanciamiento y coninamiento preventivo, medida que logró hacer evidente la pobreza, la inequidad y la desigualdad que se vive globalmente (11); así también con la investigación de Linares et al., cuando señalan que en diversos artículos se muestran una relación entre la mala calidad del aire y la infección por COVID-19; hasta el punto que la Universidad de Harvard publicó un estudio realizado en Estados Unidos sobre la posible relación entre la exposición a partículas finas (PM2,5- partículas de diámetro aerodinámico inferior a 2,5 micras) y la mortalidad por COVID-19 (12). Además de acuerdo a tabla 4 , de este estudio otro $59 \%$ responde que la población de zona de vivienda urbana es la que más se contagia de la COVID - 19; no obstante Issn et al., (13), mencionan que la pandemia golpeará con mayor fuerza a personas más vulnerables, muchas de ellas viviendo en asentamientos informales y barrios marginales en las ciudades debido a que estas áreas están densamente pobladas, con sistemas de transporte público masificados, poca o ninguna gestión de residuos, ausencia de servicios básicos y viviendas deficientes, igualmente Rodríguez considera que los elementos que interaccionan en el espacio definen el estado de salud de la población, por tanto una enfermedad afecta más a un grupo de población de acuerdo a la interacción del ser humano, el entorno, el proceso de salud y la enfermedad por tal sentido las persona más vulnerables viven en zona urbana (14). De otro resultado, un $49 \%$ considera que el aire es el elemento contaminante que contribuye al contagio 
de la COVID - 19 en la población, lo que corrobora el estudio del Comité de Salud Medio Ambiental, quienes señalan que la pandemia sanitaria mundial por Sars-CoV2 tiene una fuerte relación con la salud de nuestros ecosistemas, siendo un contaminante biológico medioambiental del aire, que se concentra y deposita durante horas en los espacios interiores; por ello la aparición de rebrotes vinculados a la contaminación del aire interior hicieron saltar alarmas de contagio (15). Además las nuevas evidencias sugieren que la contaminación atmosférica agrava los impactos del virus en la salud, haciendo que las personas sean más vulnerables a la COVID-19 y contribuye a su propagación (16).

Finalmente, es importante indicar que si la COVID-19 se propaga a nivel de Latinoamérica, es importante mantener la adopción de algunas sencillas medidas de precaución, como el distanciamiento físico, llevar mascarilla, ventilar bien las habitaciones, evitar las aglomeraciones, lavarse las manos y, al toser, cubrirse la boca y la nariz con el codo flexionado o con un pañuelo; incluyendo mejoras de la calidad del aire hasta un momento inimaginable con grandes beneficios para el medioambiente, la calidad del aire y disminución en la contaminación ambiental evidente, implementándose políticas de estado de sembrar un árbol.

Como principal limitación del presente estudio es que la muestra abarca distintos tipos de población de diferentes países de Latinoamérica, a pesar de ello las conclusiones generales nos animan a continuar profundizando esta investigación. Por otro lado, al tratarse de un cuestionario anónimo y electrónico, se asume el sesgo de alguno de los encuestados.

En conclusión, nuestros resultados revelan que los factores que contribuyeron al incremento de pacientes infectados por el COVID-19 en las diferentes familias de Latinoamérica, siendo los factores de bioseguridad los que contribuyen al contagio de la población, respondieron un $43 \%$ que podría ocasionarse por no emplear mascarilla y protector facial, así también como factores culturales un $55 \%$ consideran que se contagian por no respetar normas, igualmente en cuanto a factores ambientales un $74 \%$ señalan que la contaminación ambiental tiene que ver con el aumento del COVID - 19, otro $59 \%$ indican que la población de zona de vivienda urbana es la que más se contagia y el aire es el elemento contaminante que contribuye al contagio de la COVID - 19 en la población; por tanto, sería necesario desarrollar estrategias para reforzar los procedimientos de prevención respecto a contagio, adecuando las medidas de protección apropiadas; así como reforzar la adherencia a la higiene de manos, y todas medidas de bioseguridad.

\section{REFERENCIAS BIBLIOGRÁFICAS:}

1. Aguilar F. Desafíos para el manejo y detección de pacientes con COVID-19 en Latinoamérica. Rev Exp Med. 2020; 6(1): 43-50. DOI: https://doi. org/10.37065/rem.v6i1.436

2. Infosalus. ¿Cuáles son los factores que permitieron la expansión del COVID-19 a nivel mundial? Infosalus; 2020. (Citado el 12 de enero del 2021). Disponible en: https://www.infosalus.com/saludinvestigacion/noticia-cuales-son-factorespermitieron-expansion-covid-19-nivelmundial-20200518163636.html

3. Pighi P. Coronavirus en Perú: 5 factores que explican por qué es el país con la mayor tasa de mortalidad entre los más afectados por la pandemia. BBC; 2020. (Citado el 12 de enero del 2021). Disponible en: https:// www.bbc.com/mundo/noticias-americalatina-53940042

4. STATISTA. Estadísticas sobre El coronavirus (COVID-19) en América Latina. STATISTA; 2020. (Citado el 12 de enero del 2021). Disponible en: https://es.statista.com/estadisticas/1105121/numerocasos-covid-19-america-latina-caribe-pais.

5. Caicoya M. El papel de las mascarillas en el control de la epidemia de COVID-19. J Healthc Qual Res. 2020;35(4):203-205. doi: 10.1016/j.jhqr.2020.05.001

6. Armando J. Uso razonado de protectores faciales en la pandemia Covid-19: un enfoque bio-socio-cultural. Sonora: El Colegio de Sonora; 2020. (Citado el 12 de enero del 2021)Disponible en https://www.colson. edu.mx/promocion/img/Haro Uso $\% 20$ protectores\%20faciales\%20Covid-19.pdf

7. Gabriela A, Arroyo C, María F, Matías C. Uso de desinfectantes en tiempos de COVID-19. Milen Cienc y arte. 2020; 16:13-5. DOI: https://doi.org/10.3390/ ijerph17093326

8. Programa sobre Saneamiento e Higiene UNICEF. La importancia del lavado de manos en tiempos de COVID-19. Programa sobre Saneamiento e Higiene UNICEF ; 2020. (Citado el 12 de enero del 2021). Disponible en: https://www.cruzroja.gt/noticias/ la-importancia-del-lavado-de-manos-en-tiempos-decovid-19/.

9. Jara C. Diseño de campaña social para evitar el desacato a las normas impuestas por el Covid 19 en la ciudad de Lima Metropolitana. Tesis de Bachiller en Arte. Lima: Universidad San Ignacio de Loyola;2020. (Citado el 12 de enero del 2021). Disponible en: http://repositorio.usil.edu.pe/handle/ 
USIL/10887

10. Pichihua P. Identificando las complicidades urbanas del COVID-19 en Latinoamérica. El caso de las ciudades peruanas. Bitácora Urbano Territ. 2021;31 (2):157-71.

11. Arango R, Caraza D, Vargas E. Infección por COVID-19, una mirada a los factores ambientales relacionados con la pandemia. Nova. 2020;18(35): 101 - 105. DOI: https://doi.org/10.22490/24629448. 4193

12. Linares C, Diaz J, Plaza J, Molina P. ¿Es la contaminación atmosférica un factor de riesgo en COVID-19?,Madrid:InstitutodeSaludCarlos III;2020. (Citado el 12 de enero del2021). Disponible en: https:// w w w . con p r u e ba.es / s i tes/defa $1 \mathrm{t} /$ files/noticias/2020-05/ES\%20LA \%20CONTA MINACI $\%$ C3\%93N\%2ATMOSF\%C3\%89RICA $\%$ 20UNA $\% 20$ FACTOR \%20DE\%20RIESGO $\% 20$ EN\%20COVID-19.pdf

13. Issn U, Echeverr A. Globalización, urbanización y salud: Impactos de la COVID-19. Arquit y Urban. 2020;41(3):6-16.
14. Rodríguez C. La Geografía del COVID-19. Cent Ciencias Desarro Reg . 2020;3:1-4.

15. Comité de Salud Medio Ambiental. Covid19 Un Contaminante Que Flota En El Aire. Madrid: Comité de Salud Medio Ambiental ; 2020. (Citado el 12 de enero del 2021). Disponible en: https://www.aeped. es/sites/default/files/covid19flotaenelaire212.pdf.

16. Banco Mundial. Contaminación atmosférica: confinada pero no detenida por la COVID-19. Washington DC: Banco Mundial; 2020. (Citado el 12 de enero del 2021). Disponible en: https:/www. bancomundial.org/es / new s / i m mersivestory/2020/07/01/air-pollution-locked-down-bycovid-19-but-not-arrested.

Recibido: 10/02/2021 Aceptado: 29/05/2021 